UCRL-JC-128091

PREPRINT

\title{
The Effect of Impurities and Stress on the Damage Distributions of Rapidly Grown KDP Crystals
}

\author{
M. Runkel, M. Yan, J. De Yoreo, N. Zaitseva
}

This paper was prepared for submittal to the 29th Annual Boulder Damage Symposium

Boulder, $\mathrm{CO}$

October 6-8, 1997

December 20, 1997

This is a preprint of a paper intended for publication in a joumal or proceedings. Since changes may be made before publication, this preprint is made available with the understanding that it will not be cited or reproduced without the permission of the author. 


\section{DISCLAIMER}

This document was prepared as an account of work sponsored by an agency of the United States Government. Neither the United States Government nor the University of California nor any of their employees, makes any warranty, express or implied, or assumes any legal liability or responsibility for the accuracy, completeness, or usefulness of any information, apparatus, product, or process

disclosed, or represents that its use would not infringe privately owned rights. Reference herein to any specific commercial product, process, or service by trade name, trademark, manufacturer, or otherwise, does not necessarily constitute or imply its endorsement, recommendation, or favoring by the United States Government or the University of California. The views and opinions of authors expressed herein do not necessarily state or reflect those of the United States Government or the University of California, and shall not be used for advertising or product endorsement purposes. 


\title{
The effect of impurities and stress on the damage distributions of rapidly grown KDP crystals
}

\author{
Mike Runkel, Ming Yan, Jim DeYoreo and Natasha Zaitseva \\ Lawrence Livermore National Laboratory \\ P. O. Box 808, L-250 \\ Livermore, CA 94550
}

\begin{abstract}
Development of high damage threshold, $50 \mathrm{~cm}$, rapidly grown $\mathrm{KD} * \mathrm{P}$ frequency triplers for operation on the National Ignition Facility(NIF) in the $14 \mathrm{~J} / \mathrm{cm}^{2}, 351 \mathrm{~nm}, 3 \mathrm{~ns}$ regime requires a thorough understanding of how the crystal growth parameters and technologies affect laser induced damage. Of particular importance is determining the effect of ionic impurities (e.g. $\mathrm{Cr}^{3+}$, $\left.\mathrm{Fe}^{3+}, \mathrm{Al}^{3+}\right)$ which may be introduced in widely varying concentrations via the starting salts. In addition, organic particulates (e.g. Lucite and Teflon) can contaminate the solution as leachants from growth platforms or via mechanical ablation. Mechanical stresses in the crystals may also play a strong role in the laser-induced damage distribution (LIDD), particularly in the case of large boules where hydrodynamic forces in the growth tank may be quite high.

In order to investigate the effects of various impurities and stresses on laser damage we have developed a dedicated, automated damage test system with diagnostic capabilities specifically designed for measuring time resolved bulk damage onset and evolution. The data obtained makes it possible to construct characteristic damage threshold distributions for each sample. Test results obtained for a variety of KDP samples grown from high purity starting salts and individually doped with Lucite and Teflon, iron, chromium and aluminum show that the LIDD drops with increasing contamination content. The results also show that solution filtration leads to increased damage performance for undoped crystals but is not solely responsible for producing the high LIDDs required by the NIF. The highest LIDD measured on a rapidly grown sample indicate that it is possible to produce high damage threshold material using ultrahigh purity, recrystallized starting salts, continuous filtration and a platform designed to minimize internal stress during growth.
\end{abstract}

Keywords: KDP, laser damage distributions, impurities, bulk laser damage

\section{INTRODUCTION}

The current interest in producing high damage threshold $\left(\mathrm{D}_{\mathrm{x}} \mathrm{H}_{1-x}\right)_{2} \mathrm{PO}_{4}(\mathrm{KD} * \mathrm{P}, \mathrm{DKDP})$ crystals for use on the National Ignition Facility (NIF) is being driven by maximum fluence requirements for third harmonic generation (THG). The current maximum fluence specification for triplers is $14.3 \mathrm{~J} / \mathrm{cm}^{2}$ in a $351 \mathrm{~nm}, 3 \mathrm{~ns}$ pulse. Data from LLNL's extensive database ${ }^{1}$ indicate that even the best samples of $K D^{*} \mathrm{P}$ cannot survive these fluences without laser preconditioning. The need for laser preconditioning, however, does not preclude the need for triplers with high unconditioned (S/1) thresholds. The database also shows that the average level of conditioning for the best $\mathrm{KD}^{*} \mathrm{P}$ samples is $1.5 \mathrm{X}$. So, to achieve maximum performance and lifetime from NIF triplers material with the highest possible damage thresholds is desirable.

As part of LLNL's extensive effort to produce high damage threshold KDP and KD*P crystals for the NIF by rapid growth techniques, we have done numerous phenomenological experiments to develop an understanding of the factors involved in crystal growth and post-growth processing and the role they play in laser conditioning and damage ${ }^{2-5}$. These efforts have concentrated on understanding the role of discrete bulk inclusions ${ }^{2}$, refractive index variations $s^{3}$, and $\mathrm{UV}$ absorption variations due to the incorporation of ionic impurities into the lattice ${ }^{3,4}$. Numerous techniques have been developed to control these parameters during and after crystals growth. They include the use of highest purity starting salts, filtration of solutions prior to and during growth (continuous filtration) and application of post-growth processes such as thermal annealing and laser conditioning.

In order to determine which of the many growth and processing parameters affect the laser damage threshold, a dedicated test system was constructed to provide consistent, rapid, automated damage testing of KDP test samples. Because of the large parameter space we wish to map, a basic requirement of the system was high sample throughput. In addition, the statistical nature of laser damage also demanded rapid testing of hundreds of sites per sample. 


\section{THE ZEUS AUTOMATED DAMAGE TEST SYSTEM}

The ZEUS ${ }^{6}$ damage test facility was originally built in 1995 in response to increased damage testing demands resulting from the NIF project. It is a frequency tripled, 2 Joule Nd:YAG laser operating at $10 \mathrm{~Hz}$ with a $7.6 \mathrm{~ns}, 30$ pulse. It is capable of delivering up to $100 \mathrm{~J} / \mathrm{cm}^{2}$ of $3 \omega$ light in a 700 micron (FW@ $1 / \mathrm{e}^{2}$ ) spot. Initially, the sole damage detection diagnostic consisted of pre- and post-exposure visual inspection of the test site via Nomarski/darkfield illumination at $100 \mathrm{X}$ magnification. For studying the role of discrete bulk scatterers as well as refractive index variations in laser damage ${ }^{25}$ a high spatial resolution, 14 bit CCD camera and $527 \mathrm{~nm} \mathrm{Nd:YLF} \mathrm{probe} \mathrm{laser} \mathrm{was} \mathrm{subsequently} \mathrm{added.} \mathrm{During} \mathrm{the} \mathrm{course} \mathrm{of} \mathrm{these}$ experiments it became apparent that LLNL's traditional methods of S/1 (600 shots/site @ fixed fluence) and R/1 (600 shots/site with ramped fluence) damage testing could not provide statistically meaningful indicators of damage performance due to the small number of sites tested. To correct this problem we installed hardware and developed software to automate the testing process using the systems developed by Hue et. al. ${ }^{7,8}$ as a guide. Because of the importance of conditioning in our development work, we decided to emphasize $R / 1$ conditioned testing in automated tests. This had the added benefit of being able to test every site to failure, which would give a more complete statistical description of damage performance than any $\mathrm{S} / 1$ test method.

Modifications to ZEUS included installation of computer control on the variable attenuator waveplate rotation stage (energy control), sample translation (XY) stages and beamline shutters. A data acquisition cand was used to record voltage readings from the damage detection diagnostic which consisted of a $30 \mathrm{~mW}$ HeNe probe laser and photomultiplier tube. This arrangement detects changes in the forward bulk scatter intensity from the crystal at approximately 45 degrees (see Figure 1.). The diagnostic allows the evolution of bulk scatter events at a test site to be reconded in real time as a function of the peak fluence in an individual laser pulse. The automation software allows user control over the number of sites tested, the sample grid (site spacing), the number of pulses per site and the fluence ramp rate $\left(\mathrm{J} / \mathrm{cm}^{2} / \mathrm{s}\right)$. In addition, voltage thresholds for damage detection and test termination as well as site acceptability are user controlled.

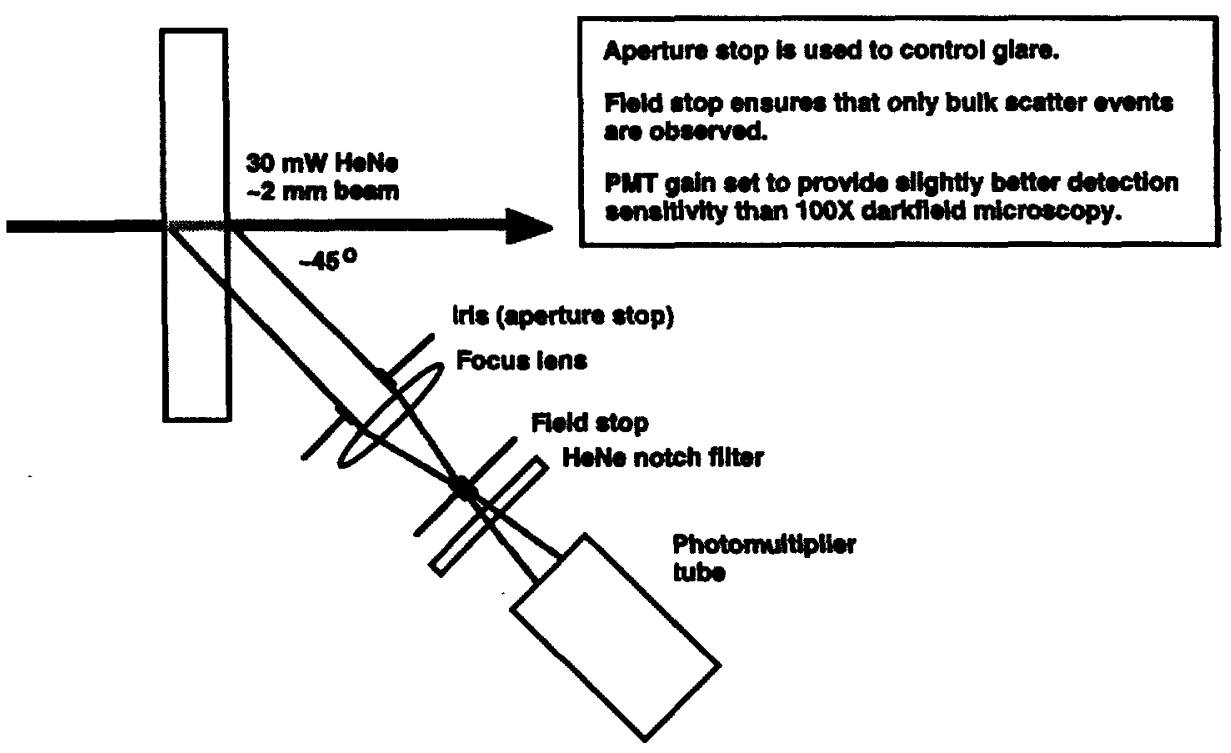

Figure 1. The Zeus bulk scatter diagnostic. The diagnostic detects changes in the bulk scatter of the test sample during irradiation. The aperture stop is used to control glare while the field stop is used to control the volume seen by the PMT. In this manner surface scatter can be virtually eliminated. The PMT gain is set empirically to provide comparable detection to $100 \mathrm{X}$ darkfield microscopy.

Our standard automated KDP damage test consists of exposing one hundred individual test sites to ramped fluence pulses until catastrophic damage occurs. For the 700 micron damage spot a grid spacing of $2 \mathrm{~mm}$ is used. Each test site is ramped from zero fluence up to damage at $0.5 \mathrm{~J} / \mathrm{cm}^{2} / \mathrm{s}$. The beam diameter for the $30 \mathrm{~mW}$ HeNe probe laser is set to approximately $2 \mathrm{~mm}$. 
This spot diameter ensures approximately uniform illumination of the damaged volume without interacting appreciably with previously tested sites. A scatter trace of typical events is shown in Figure 2. After sample irradiation the individual traces are analyzed to determine the earliest onset of damage at each site as shown in the figure. The associated onset fluences are recorded, sorted in ascending order and plotted. The sorted data can be interpreted as a failure probability curve. When the failure probability data is plotted versus the failure fluence the result is a characteristic " $S$ " curve for the sample. The derivative of this curve is essentially the damage distribution of the sample. As shown in Figure 3 and later in Figure 5., appropriate binning of the distribution data can yield information regarding the number of damage mechanisms involved.

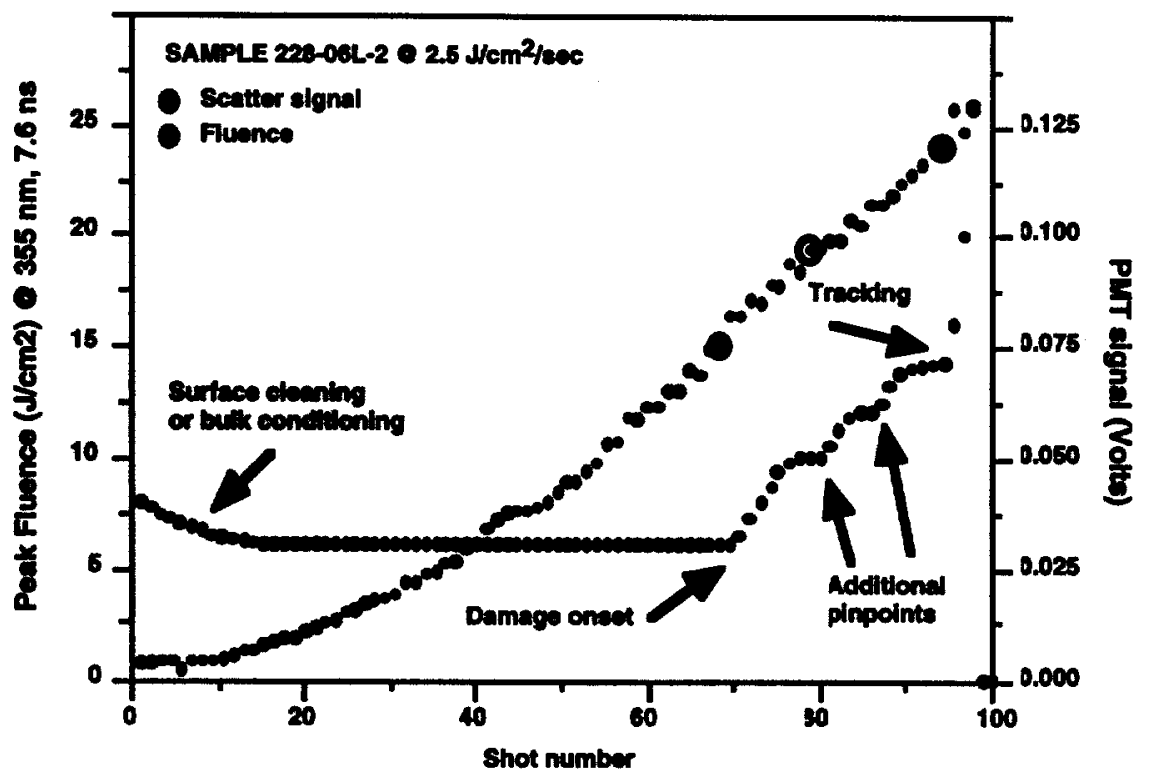

Figure 2. Typical scatter response from the bulk scatter diagnostic as a function of incident fluence. Features include surface cleaning/bulk conditioning, stabilization of the scatter signal until the formation of the first pinpoint, the formation of additional pinpoints up to catastrophic damage (tracking).

\section{SAMPLES AND TEST RESULTS}

Once the appropriate automation handware and software had been integrated onto the damage test station, we carried out a matrix of tests designed to address the effect of numerous parameters on damage as mentioned above. The first issue which regarded the relationship between the old $R / 1$ and new automated test methods. After this we tested a number of rapidly grown crystals to assess the impact of starting salt purity, continuous filtration of solution, cationic impurity concentration and stress due to platform shape.

\subsection{Testing of KDP crystal "vintages"}

As far as LLNL ICF laser systems are concerned, the evolution of KDP/KD*P crystal growth technology can be traced through four distinct eras or vintages. The first is for crystals grown prior to construction of the NOVA laser (circa 1986, known as "pre NOVA') The second is for crystals grown after NOVA in the period from 1987-1990 ("post NOVA"), the third vintage is for crystals grown explicitly for the Beamlet laser (1994, "Beamlet vintage"). The first three vintages concern themselves exclusively with crystals grown conventionally (i.e. growth rates of $-1 \mathrm{~mm} /$ day, pyramidal sector only.) The last vintage refers to rapidly grown KDP/KD*P crystals using technology currently under development at LLNL. Rapid growth crystals are grown at nominal rates of $\sim 10 \mathrm{~mm} /$ day and contain both prismatic and pyramidal sectors. Because of the considerable cost savings and risk reduction potential offered by rapid growth crystals, they are currently considered to be the baseline growth method for crystals to be used on NIF as Pockel's cells and second and third harmonic generators. 


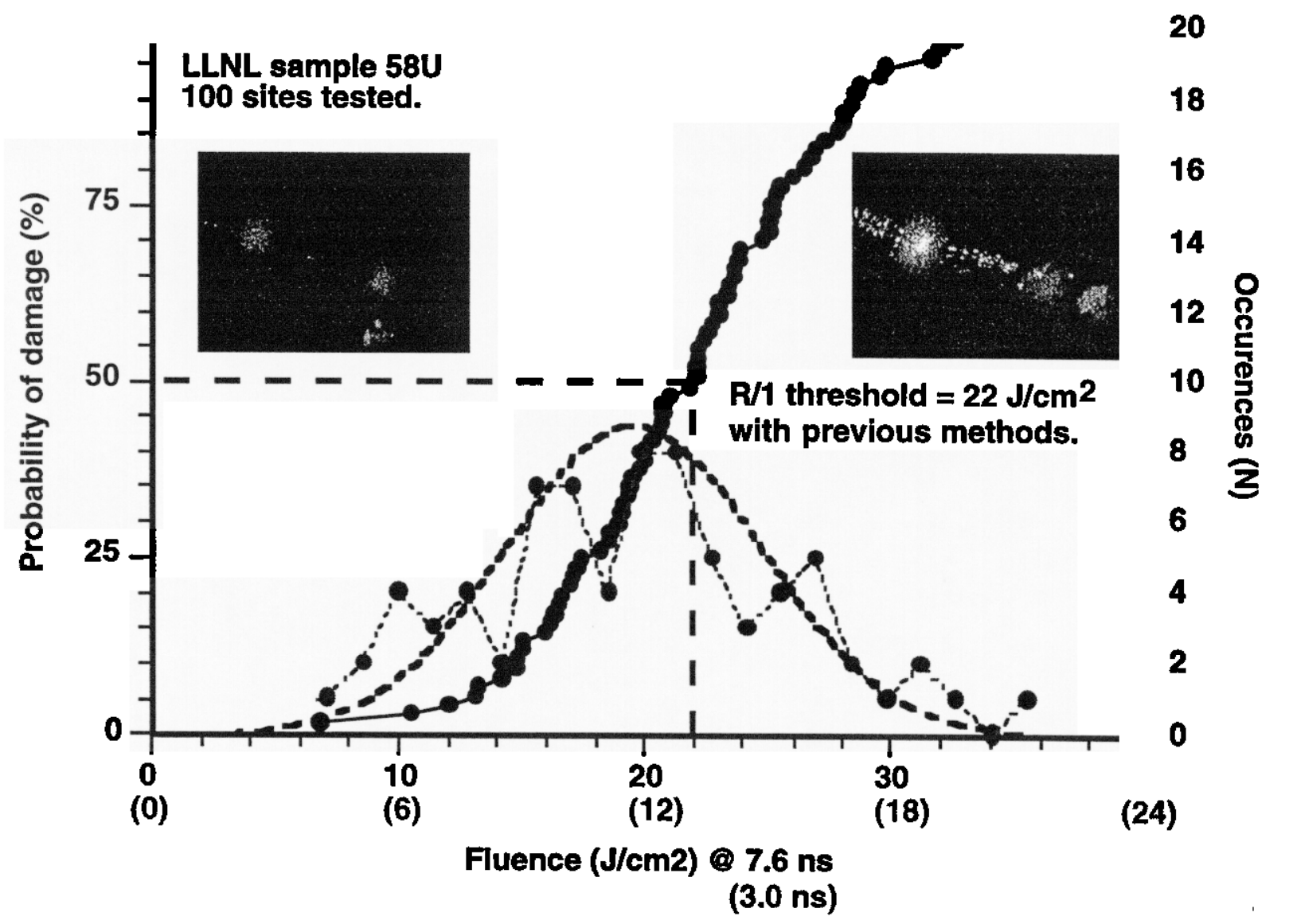

Figure 3. The characteristic damage probability curve and associated distribution for LLNL rapidly grown sample 58U. The upper left contains a photograph of a single damage pinpoint typical of the low fluence morphology while the upper right photo shows the tracking morphology common to failures occurring at high fluence. A normal distribution offers good fit to the general shape of the distribution. The data is scaled between $3 \mathrm{~ns}$ and $7.6 \mathrm{~ns}$ via $\tau^{0.5}$.

Figure 4 shows the LIDDs for seven crystals spanning the four vintages. Included in the set are samples 228-06L (pre NOVA KDP), 282-74 (post NOVA KDP) which represent typical samples, LL6-56 (KD*P) and 311-LG1 (KDP) which represent the highest damage threshold of crystals their type ever tested at LLNL. Rapid growth is represented by KDP samples 115, RG1 and 214. Sample 115 represents the first rapid growth boule produced with continuous ultrafiltration of the growth solution. Sample RG1 represents material from the LLNL's first rapidly grown $50 \mathrm{~cm}$ boule (grown without continuous filtration) and sample 214 is the highest damage threshold, rapidly grown crystal grown to date. It was produced from ultrahigh purity starting salts, continuous filtration and growth of the boule on a platform designed to reduce internal stress in the crystal.

As may be expected, the lowest distribution is yielded by the pre-NOVA sample 228-06L. Post-NOVA sample 282-74 and LLNL rapid grown sample 115 are not significantly better. LLNL KDP sample RG1 shows the same low fluence damage behavior but is more damage resistant at higher fluences. The conventionally grown KD*P sample LL6-56 and rapidly grown KDP sample 214 exhibit virtually identical damage distributions while conventionally grown KDP 311 shows the highest thresholds for KDP measured at LLNL. The circles shown on the curves for samples 282-74, LLNL 115, LL6-56 and 311-LG1 are threshold fluences from previous (nonautomated) $R / 1$ tests. The large variation in the damage probabilities associated with the old $R / 1$ threshold fluences indicates the inadequacy of the old test method to be a reliable predictor of damage performance. The variations stem from the small number of points sampled (typically 10 or less) in the old method. In particular, the old R/1 test data for LL6-56 does not even fall on the damage distribution. Numerical data for these probability curves is given in Table 1 at the end of section 3.3 . 


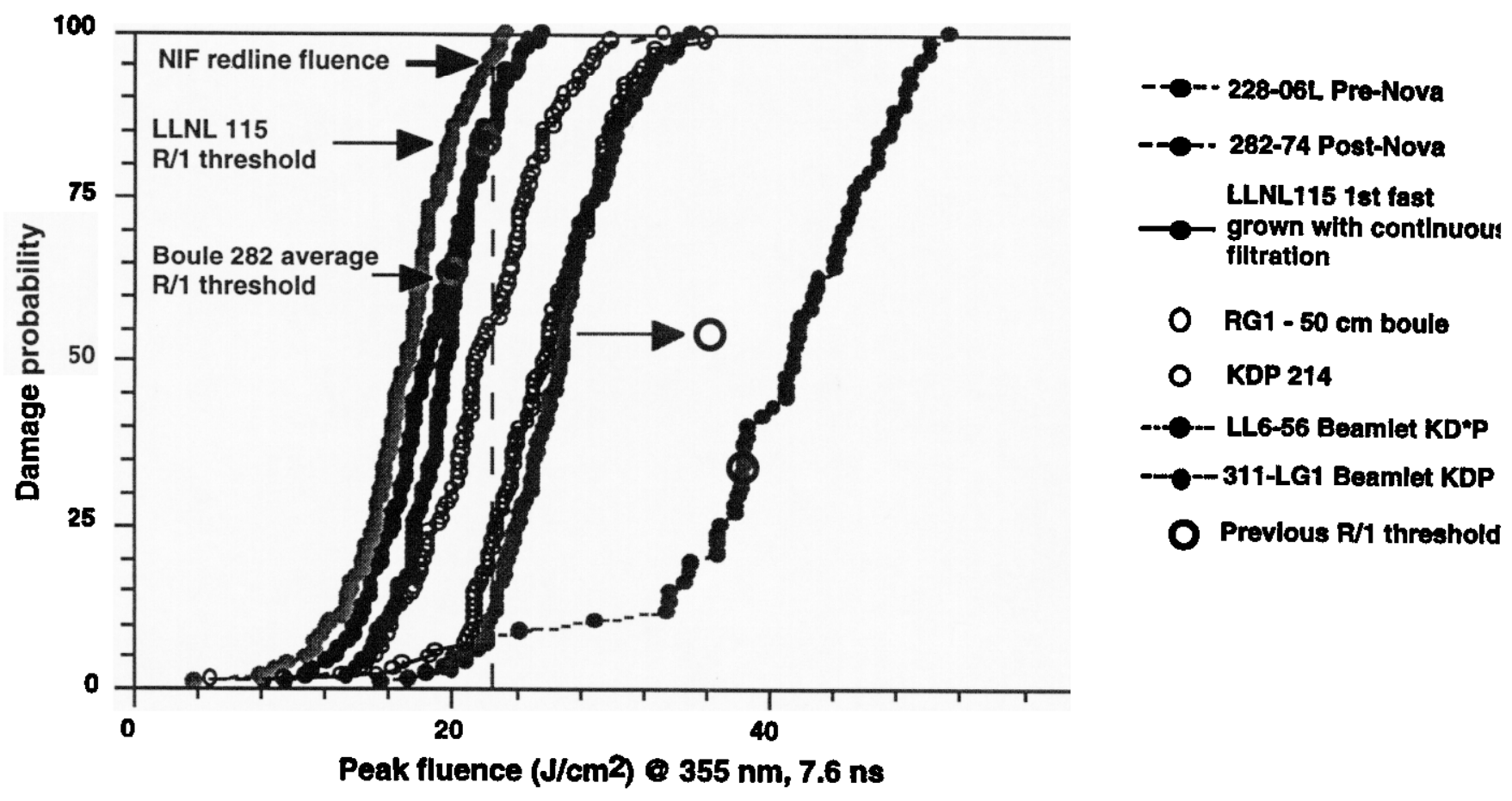

Figure 4. Damage distributions for seven historically significant KDP/KD*P crystals compared to the NIF redline fluence. Samples are listed in order of increasing damage performance.

As shown in Figure 6, when the overall sample performance increases the damage distribution histogram will have more than one peak (sample 214). This can be attributed a reduction in the density of defects which damage at low fluence. This allows test sites to reach higher fluences where additional damage mechanism can be activated. It is evident from the data that as the crystals increase in performance the toe of the curve starts at higher fluence as well. Even in the best crystals produced to date (e.g. 311), however, this class of defects has not been entirely eliminated. We discuss the implications for NIF performance in a separate section below.

\subsection{The effect of particulate contamination on damage distributions}

A three sample set was grown to assess the impact of large particulate contamination on damage. It consisted of samples 149,154 and 171 . The solution of sample 149 was prefiltered using 0.02 micron filtration and then intentionally doped with a concentration of Lucite and Teflon particulates to simulate the type of contamination which might arise from mechanical ablation in the growth tank. The particles were obtained by shaving material into the growth solution. Enough material was added so that the solution took on a slight milky tint. Sample 154 was grown from unfiltered, undoped solution which was more representative of the normal growth parameters, while sample 171 was grown from continuously filtered solution into which a bubble stream of dry nitrogen was injected. The purpose of this experiment was to assess the impact of very small bubbles which may form in the solution due to turbulence. Samples 149 and 154 showed a high number of visible inclusions due to the contamination. Sample 171 on the other hand did not show any visible contamination or inclusions. The results of testing are shown in Figure 5.

Unfiltered sample 154 exhibits the poorest overall damage performance of any crystal tested to date. Sample 149 is only slightly better at higher fluences, but exhibits the same onset while 171 shows damage behavior comparable with the benchmark sample 115. These results clearly indicate the need for rudimentary solution cleanliness prior to and during growth. As a direct result of these experiments we investigated the degree of solution cleanliness needed to achieve high damage distributions for KDP. The single most important/effective means of achieving and maintaining solution cleanliness is via filtration. 


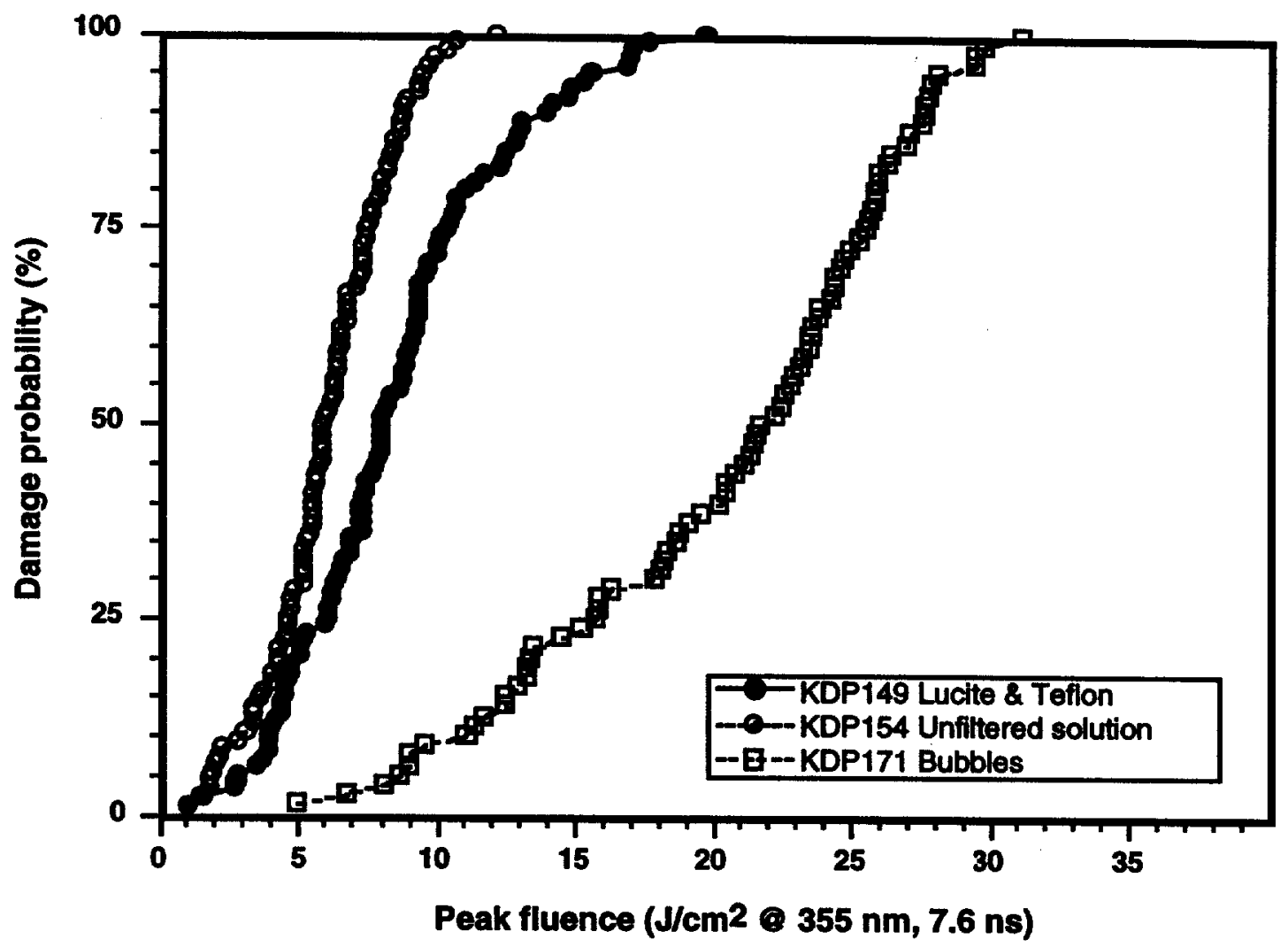

Figure 5. Damage probability curves for samples 149,154 and 171 . The growth solutions were deliberately doped with Lucite and Teflon particulates (149), bubbles(171) or left unfiltered before and during growth (154).

\subsection{The effect of continuous filtration on damage distributions}

Continuous filtration of KDP growth solutions has been available since its development in 1989 at LLNL by Montgomery and Milanovich'. Published data indicated that continuous filtration could be used to dramatically increase the damage thresholds of KDP at $1064 \mathrm{~nm}$ as well as a giving a substantial rise at $355 \mathrm{~nm}$. Encouraged by Montgomery and Milanovich's results for conventionally grown KDP we used a continuous filtration system to grow sample 115 . The solution was withdrawn from the tank at approximately $\sim 1.0 \mathrm{~V} / \mathrm{min}$, heated prior to filtration to reduce the risk of spontaneous nucleation, then filtered with a $\mathbf{0 . 0 2}$ micron filter. The solution was cooled to growth temperature prior to being pumped back into the tank. As shown in Figure 4, its damage performance was only slightly better at low fluence than the typical post-NOVA crystal. Since this was our first attempt at using continuous filtration on a rapid growth crystal, it is likely that optimal filtration parameters were not being used which resulted in the low LIDD of the crystal. This result, coupled with the fact that we have grown unfiltered crystals which were better than 115 (unpublished data) lead us to question the central role played by continuous solution filtration in increasing damage performance, as claimed by Montgomery and Milanovich. Our subsequent analysis of their data did not support such a strong conclusion because there were other uncontrolled factors involved which were not mentioned in [9]. We could only conclude that it was likely that continuous filtration of growth solution contributed to high damage thresholds.

To arrive at a firm conclusion regarding the value of solution filtration we grew two samples from ultra high purity (UHP) starting salts, one (214) with continuous filtration and one without (210). The salts were obtained by accumulating numerous decapitated pyramidal tops from previously grown boules. This was done because a large number of elemental, cationic impurities are not incorporated into the pyramidal sector ${ }^{4}$ and the resulting material is very pure. In addition, a convex platform was installed in the tank for the 214 growth run. This platform was designed to reduce the stress buildup in the crystal. The solution for sample 210 , on the other hand, was prefilterd only prior to growth. Lack of continuous filtration on sample 210 lead to a high level of visible inclusions and it was expected that damage performance would be poor. The results of testing are shown in Figure 6. 


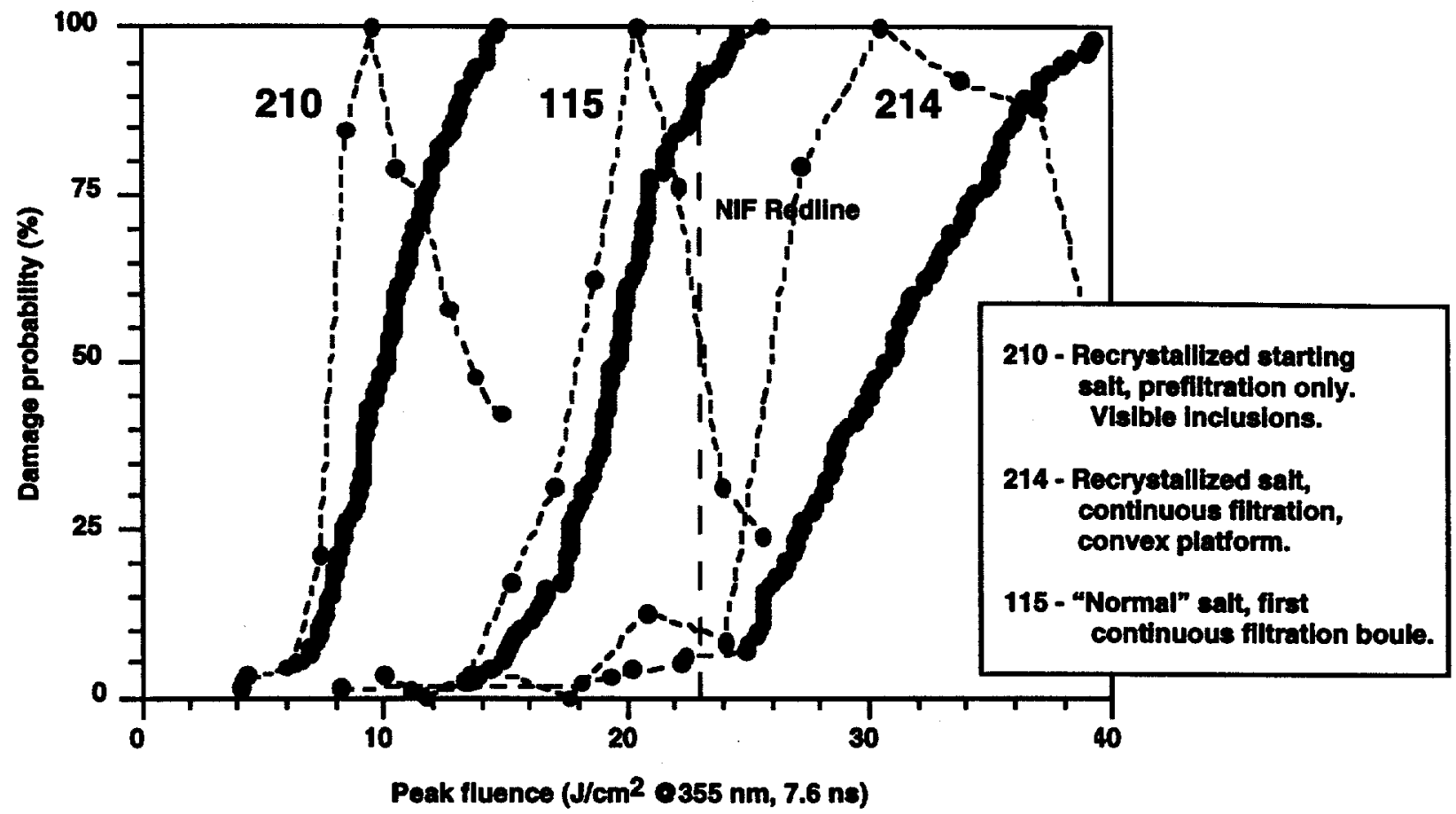

Figure 6. Test results and damage histograms for samples 214 and 210 . The samples were grown from ultra-high purity starting material with and without continuous filtration respectively. Damage performance is reduced by a factor of 3 in the contaminated sample. Contamination in sample 210 was visible to the naked eye in room light. The damage distributions for samples 115 and 214 yield good fit using normal distributions while sample 210 does not.

Despite the deviations from a well controlled single parameter experiment, several conclusions can be drawn from the data. First, the combination of UHP salts, continuous filtration and convex growth platform yielded the best damage distribution for a rapidly grown crystal to date. It is virtually identical to the performance of the best KD*P samples tested (boule LL6 material). In contrast, lack of continuous filtration, severe particulate contamination and a flat growth platform yielded nearly the worst performance of any crystal tested to date. Comparison of samples 210 and 115 , which was grown with less clean ("typical") salt, a flat platform and continuous filtration indicate the effect of solution cleanliness on damage distributions. While none of these experiments have sufficiently isolated the effect of continuous filtration on damage, they none-the-less indicate its importance to the growth process. As a result all future rapidly grown crystals will be produced with continuous filtration systems in place.

\subsection{The effect of ionic impurities on damage distributions}

Once it was determined that continuous filtration was necessary to achieve high damage threshold crystals, we sought to determine the effect of other materials which would not necessarily be filtered out of the solution. We focused on cationic species $\mathrm{Fe}, \mathrm{Cr}, \mathrm{Sb}$ and $\mathrm{Al}$ which are found with the KDP salts. We took this approach because previous studies indicated ${ }^{3}$ 10${ }_{12}$ that cations are incorporated into the prism and pyramidal sectors of KDP in different concentrations, and that these ions give rise to UV absorption bands at approximately $300 \mathrm{~nm}$. For example, we have seen that $\mathrm{Fe}$ is incorporated nearly 100 times more readily into the prism sector of KDP compared to the pyramid, and that this leads to an increase in ultraviolet optical absorption of at least an order of magnitude. We questioned whether this difference would manifest itself in the damage distributions of the material. To that end, we tested five samples whose growth solutions were deliberately doped with different ions. All the impurity species were introduced into the growth solutions as nitride compounds in the following concentrations (relative to solution): Aluminum - 3 ppm, Iron - 1 ppm, 5 ppm, Chromium - 2 ppm, Antimony $10 \mathrm{ppm}$. It must be noted at the outset that the solutions were not continuously filtered during growth. Unfortunately, this may mask any effect of the ionic impurities on the damage probability curves. 


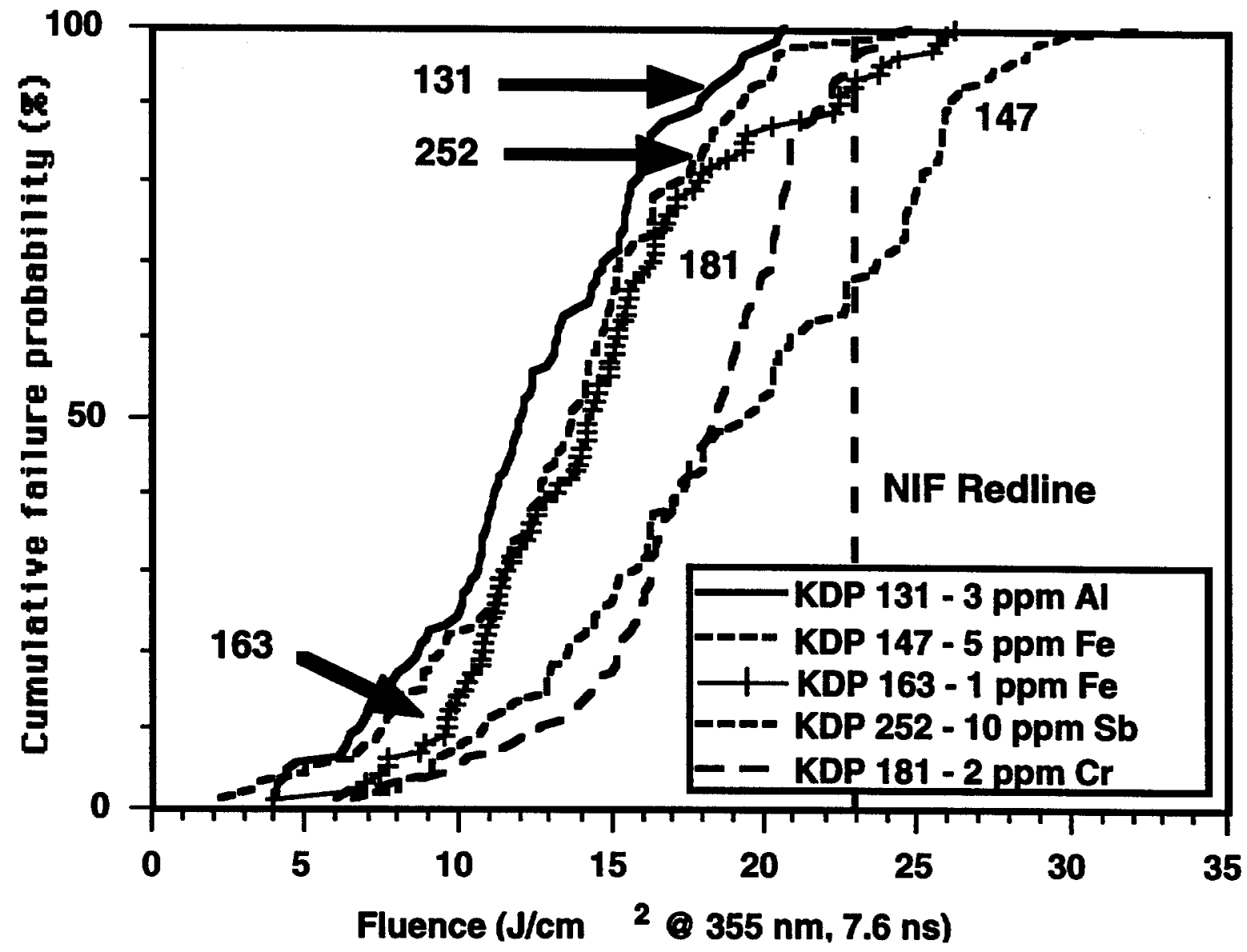

Figure 7. Damage probability curves for samples grown from cationic impurity doped solutions. These ionic impurities were chosen because they commonly occur in starting KDP powders. Ion concentrations are relative to solution.

The aluminum and antimony doped samples (131 and 252) exhibit the poorest damage behavior followed by the $1 \mathrm{ppm}$ Fe doped sample (163). The performance of theses samples is basically identical when the $\pm 15 \%$ fluence measurement uncertainty is taken into account. The chromium doped sample (181) exhibits a curve very similar to LLNL 115, while the 5 ppm doped Fe sample (147) exhibits the best damage performance of the set. Note however that none of these samples performs very well relative to the NIF redline fluence. While the data is not shown, it is worthwhile mentioning that no differences in the damage performance were observed between the prism and pyramidal sectors of any of the samples. These results have shifted the emphasis of our work in determining a cause for KDP damage to scenarios where the impurities may form phosphate based nanoclusters as in the case of $\mathrm{FePO}_{4}$. Preliminary measurements on $\mathrm{FePO}$ indicate that the nanoclusters may have adequate UV absorption to lead to damage (ca. $10^{3} \mathrm{~cm}^{-1}$ ). We hypothesize that the nanoclusters are incorporated into the prism and pyramid sectors in equal concentrations because they are large enough to be unaffected by the net charge on the surface of either growth sector. (It should be noted that these surface charge states have been implicatedin the dramatic difference in ionic impurity concentration between sectors.) This hypothesis accounts for the observation that the LIDD is unaffected by crystal sector. Currently there are plans to grow samples intentionally doped with iron and aluminum phosphates to test this hypothesis. In addition, further experiments are required with continuously filtered samples to make definitive conclusions regarding the role of ionic impurities. Table 1 summarizes the damage test data of samples discussed in this work. 
Table 1. Summary of damage distribution data for all samples tested. Fluence values are for the minimum, maximum, average and standard deviation of the damage distribution and are for $7.6 \mathrm{~ns}$ pulses. Conversion to $3.0 \mathrm{~ns}$ pulses via $\mathrm{t}^{0.5}$ scaling results in a reduction of the values by 0.63 . The three sections in the table refer to the test matrices for benchmark samples, filtration experiment samples and impurity samples.

\begin{tabular}{|c|c|c|c|c|c|c|c|}
\hline Sample & Material & $\begin{array}{l}\text { Growth } \\
\text { method }\end{array}$ & Filtration & $\begin{array}{c}\text { Damage } \\
\mathbf{F}_{\min }\end{array}$ & $\begin{array}{l}\text { tion flue } \\
\text { Q } 7.6 \mathrm{~ns} \\
\mathrm{~F}_{\mathrm{mgx}}\end{array}$ & $\begin{array}{l}\text { values }\left(J / \mathrm{cm}^{2}\right. \\
F_{\mathrm{uve}} \pm \sigma\end{array}$ & Comments \\
\hline 228-06L & KDP & slow & None & 3.9 & 23.6 & $17.1 \pm 3.5$ & Pre-NOVA \\
\hline $282-74$ & KDP & slow & Continuous & 9.6 & 25.5 & $18.5 \pm 3.4$ & Post-NOVA \\
\hline LLNL 115 & KDP & rapid & Continuous & 8.3 & 25.7 & $19.6 \pm 2.8$ & $\begin{array}{l}\text { First continuous filtered rapid, } \\
\text { growth crystal }\end{array}$ \\
\hline RG1 & KDP & rapid & prefiltered & 5.0 & 34.9 & $22.9 \pm 4.6$ & First $50 \mathrm{~cm}$, rapid growth boule \\
\hline KDP 214 & KDP & rapid & continuous & 9.4 & 36.2 & $25.7 \pm 4.3$ & $\begin{array}{l}\text { High purity salts, convex } \\
\text { platform }\end{array}$ \\
\hline LLG-56 & $\mathrm{KD} * \mathrm{P}$ & slow & continuous & 15.6 & 35.2 & $26.7 \pm 3.7$ & Best KD*P \\
\hline 311-LG1 & KDP & slow & continuous & 17.4 & 51.3 & $40.2 \pm 7.7$ & Best KDP \\
\hline KDP 149 & KDP & rapid & prefiltered & 1.0 & 19.7 & $8.5 \pm 3.8$ & Lucite and Teflon added \\
\hline KDP 154 & KDP & rapid & none & 1.2 & 12.2 & $6.1 \pm 2.2$ & Unfiltered solution \\
\hline KDP 171 & KDP & rapid & prefiltered & 5.0 & 31.3 & $20.5 \pm 6.3$ & Bubbles injected in solution \\
\hline KDP 210 & KDP & rapid & prefiltered & 4.2 & 14.8 & $10.2 \pm 2.3$ & UHP salts, flat platform \\
\hline KDP 131 & KDP & rapid & prefiltered & 4.1 & 20.8 & $12.4 \pm 4.1$ & Solution doped with $3 \mathrm{ppm} \mathrm{Al}$ \\
\hline KDP 147 & KDP & rapid & prefiltered & 6.6 & 32.3 & $19.4 \pm 6.0$ & Solution doped with $5 \mathrm{ppm} \mathrm{Fe}$ \\
\hline KDP 163 & KDP & rapid & prefiltered & 4.0 & 26.2 & $14.7 \pm 4.8$ & Solution doped with 1 ppm Fe \\
\hline KDP 181 & KDP & rapid & prefiltered & 6.0 & 25.1 & $18.0 \pm 3.8$ & Solution doped with 2 ppm $\mathrm{Cr}$ \\
\hline KDP 252 & KDP & rapid & prefiltered & 2.3 & 24.9 & $13.6 \pm 4.5$ & Solution doped with $10 \mathrm{ppm} \mathrm{Sb}$ \\
\hline
\end{tabular}

\section{DISCUSSION}

\subsection{Regions of damage performance}

Significant discussion of the individual test matrices for the effects of large particulates, filtration and ionic impurities has been given in the preceding sections. Close inspection of Figure 4 will show that there are at least three regimes of damage associated with the curves seen in this work. In order to illustrate this point more clearly Figure 8 shows three curves which fall in the different regimes. Region I denotes samples whose damage behavior is completely dominated by large inclusions. This is typified by sample 149 which was doped with large particles of Lucite and Teflon which are strong absorbers in the UV. This region also includes conventionally grown samples without filtration from the pre-NOVA vintage. Cleaning the samples of these large defects through the use of high purity starting salts, pre-and continuous filtration of the growth solutions raises damage performance into region II. Here the overall damage performance is pushed upward in fluence with the reduction in large absorbers allowing a different behavior to emerge. We speculate that the difference in behavior is due to two factors. The first is a change in the absorption process between regions. There are a number of possibilities to consider such as a change in the size distribution of the absorber which changes how it couples to the laser field as well as the crystal lattice, or a change in the absorption mechanism itself i.e. from linear to two photon absorption. The second factor is the reduction in the number of defects and the coincident increase in the defect spacing. The resulting LIDD is less dominated by gross absorbers. The presence of the low fluence tail, however, indicates that some strong local absorbers remain in the material despite filtration. We expect that this tail will be present in even the most carefully prepared samples as seen with sample 311. This is plausible due to many factors involved in crystal growth such as the time lag between particulate formation in the growth tank and filtration. Cleaning the material of still more defects allows transition to region III. Because of the similar characteristics of the LIDD for regions II and III there may not be a hard transition between them. On the other hand, as materials become so defect free, the damage behavior will eventually transition to behavior typified by the 
intrinsic damage threshold of the pure material. In this regime non-linear effects such as self-focusing will be come apparent and the damage morphology will change dramatically ${ }^{15}$.

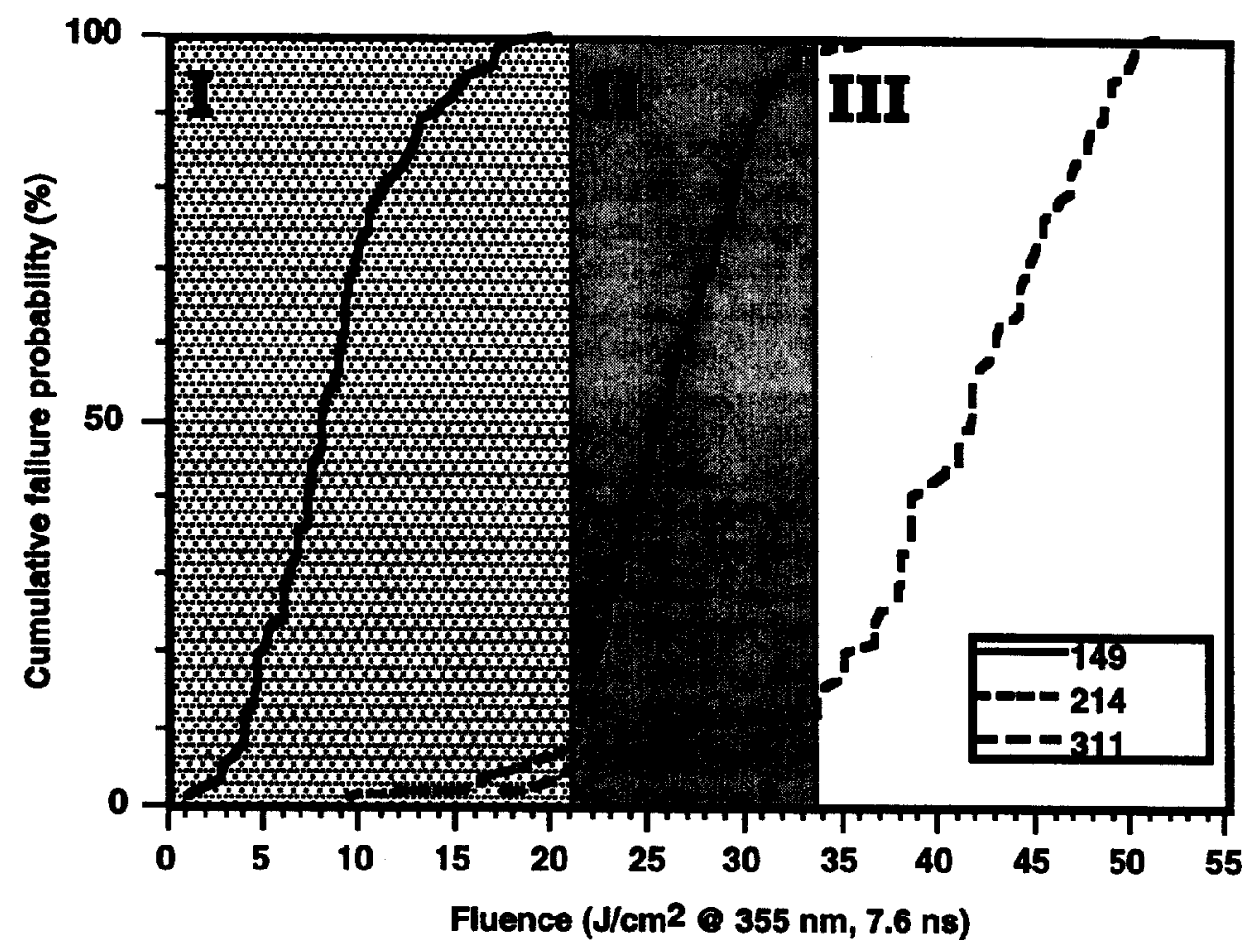

Figure 8. Cumulative damage probability curves for samples 149, 214 and 311. These samples illustrated typical behavior for samples in regions I, II and III respectively. Region I is dominated by large inclusions. As inclusion density decreases the damage fluence increases as typified by region II. Ultra-high purity samples exhibit behavior shown in region III where non-linear effects such as self focusing begin to occur. Note that despite the increases in damage performance there is still a long tail representing sites which will fail at low fluence.

\subsection{Implications for NIF}

The results shown above for the best KDP and KD*P produced to date indicate that there is small, but nonzero probability of damage at NIF redline fluences. This is indicated in the long tail characteristic of any good crystal e.g. LL6-56, KDP 214 and 311 shown in Figures 4 and 6. Numerous questions arise in interpreting these curves with regard to damage performance on NIF. We briefly discuss here methods which have been used to address this problem. Predictions of NIF THG damage performance are dependent on both the statistics of the LIDD and the distribution of fluence in the beam. Damage is likely to occur where the fluence distribution overlaps the damage distribution. Reliability theory 13.14 can be applied to histograms to calculate the failure probability for a given crystal and spatial distribution. We have performed preliminary calculations of this nature for sample 214 \& LL6-56 LIDDs as well as for sample 311. We used a model NIF spatial profile for the triplers from PROP92 (courtesy of Wade Williams). Our calculations indicate that for well conditioned samples LL6-56 and 214 there is a failure probability of approximately $2.6 \%$ In contrast the failure probability for sample 311 is $\sim 0.5 \%$. The higher failure probability is the result of a larger low fluence tail on the 214 and LL6-56 damage distributions. Because of the $\pm 15 \%$ error bar implicit in all damage measurements there is likely to be substantial variation in each of the failure probabilities given. This means that LL6-56, 214 and 311 offer equivalent damage performance as far as the NIF is concerned, this despite the fact that the majority of sites on 311 will withstand substantially higher fluence than the other two samples. These results also indicate that rapidly grown KDP will survive NIF fluences with minimal damage.

Despite the encouraging results that there will be low failure probability for NIF triplers, the reliability analysis does not give an indication of the level of damage which will occur in the triplers. To address this problem we applied the results of our 
recent OSL damage campaign ${ }^{15}$ to the model NIF THG profile mentioned above. The damage evolution model for samples LL6-56 and 214 was convoluted with the NIF profile to yield the number of pinpoints which would form in the bulk of the material. The calculations indicated that a maximum of eight (8) damage pinpoints would be formed in a crystal volume of $0.1 \times 0.1 \times 1.0 \mathrm{~cm}^{3}$, and that this would only occur at the points of highest fluence in the beam. This calculation confirms the low damage probability calculations mentioned above and gives us further confidence that rapid growth crystals with LIDDs comparable to 214 should survive NIF shots with minimal damage.

To assess the actual performance of a KD*P tripler under NIF-like conditions we mapped the $37 \mathrm{~cm}$ Beamlet tripler on the PLATO system using the bulk scatter diagnostic described earlier. The tripler crystal is from conventionally grown KD*P boule LL1. Damage test samples from LL1 compare favorably to LL6-56, and we therefore assumed that the associated LIDD for LL1 would be the same. Scatter mapping revealed that there were isolated clusters of pinpoints arising from high fluence areas in the beam. The maximum number of pinpoints detected was on the onder of 35 in a volume of $7.1 \mathrm{~mm}^{3}$ (i.e. the HeNe irradiation volume). Unfortunately, spatial profiles of the high fluence shots which may have caused this damage are unavailable for fluence distribution analysis. This precludes checking the validity of the OSL/NIF damage model under real operational circumstances. However, in spite of the fact that there is isolated, minor damage in the Beamlet tripler there are no indications from operational data that the damage has adversely impacted tripler performance.

\section{CONCLUSIONS}

In this paper we have described the role of expanded damage test capabilities being applied to the development of rapidly grown KDP and KD*P for use on the NIF. This capability was implemented because it was discovered that the statistical nature of laser damage could not be fully explored using previous $S / 1$ and $R / 1$ test methods. For KDP the current test method consists of exposing 100 sites to slowly increasing fluences until failure occurs. The evolution of damage is recorded via forward scattered visible laser light on a PMT. A characteristic damage distribution is developed by determining the failure onset for each test site and sorting them in ascending order. From this curve a histogram can be derived which gives the damage distribution.

We have tested a multitude of samples to explore the effect of various parameter variations on the damage distributions. Initially numerous samples from the various KDP vintages were tested to provide benchmarks of damage performance. It was found that the performance of conventionally grown KDP increased over time. This was attributed to improvement of growth technology. Conventionally grown KDP from boule 311 showed the highest average fluence of any crystal tested to date, but exhibited a long tail of low fluence failure probability.

After benchmarking the various crystal vintages, we tested numerous rapidly grown KDP crystals to assess the impact of continuous filtration and cationic impurities on damage performance. Samples which were intentionally doped with large particulates of Lucite and Teflon showed the lowest damage performance, indicating the need for filtration. In addition, a sample was grown with ultra high purity starting salts but without continuous filtration. It showed visible inclusions and also exhibited very poor damage performance.

Considering cationic impurities, samples were tested from crystals intentionally doped with aluminum, chromium, antimony and iron. All of the LIDDs for these samples were quite low compared to the NIF redline. None of the samples were continuously filtered and this may mask any effect of the ionic impurities on damage performance. It was noted that there were no observable differences in the LIDDs between prism and pyramidal sectors for any of the samples, indicating that impurities directly incorporated into the KDP lattice do not directly affect the damage threshold. This is despite the fact that they may be incorporated into each sector in dramatically different concentrations. From this result we are currently exploring the role of nanoclusters (particularly $\mathrm{FePO}_{4}$ ) in absorption and laser damage. We think one possible explanation for the discrete nature of KDP damage is by incorporation of nanoclusters of $\mathrm{FePO}_{4}$ in uniform concentration between prism and pyramidal sectors. Preliminary measurements indicate that the clusters could exhibit high enough absorption to lead to discrete points of laser damage as observed in KDP.

This work performed under the auspices of the U. S. Department of Energy by Lawrence Livermore National Laboratory under Contract No. W-7405-ENG-48. 


\section{REFERENCES}

1. F. Rainer, L.J. Atherton, J.J. DeYoreo, "Laser damage to production- and research grade KDP crystals," in Laser Intuced Damage in Optical Materials.SPIE Proceedings, Volume 1848, edited by H.E. Bennet et. al., 46-58, SPIE, Bellingham, WA. 1992

2. M. Runkel, B. Woods, M. Yan, J. DeYoreo, M. Kozlowski, "Analysis of high resolution scatter images from laser damage experiments performed on KDP," in Laser Induced Damage in Optical Materials, SPIE Proceedings, Volume 2714, edited by H.E. Bennet et. al., 185-195, SPIE, Bellingham, WA. 1995

3. M. Yan, J. DeYoreo, N. Zaitseva, R. Torres, "Impurity contamination in fast grown KDP," in Laser Induced Damage in Optical Materials. SPIE Proceedings, Volume 2714, edited by H.E. Bennet et. al., 196, SPIE, Bellingham, WA. 1995

4. M. Yan, R. Torres, M. Runkel, B. Woods, I. Hutcheon, N. Zaitseva, J. DeYoreo, "Impurity and laser-induced damage in the growth sectors of rapidly grown KDP crystals," in Laser Induced Damage in Optical Materials._SPIE Proceedings, Volume 2966, edited by H.E. Bennet et. al., 11-16, SPIE, Bellingham, WA. 1996

5. B. Woods, M. Runkel, M. Yan, M. Staggs, N. Zaitseva, M. Kozlowski, J. DeYoreo, "Investigation of damage in KDP using light scattering techniques," in Laser Induced Damage in Optical Materials,_SPIE Proceedings, Volume 2966, edited by H.E. Bennet et. al., 20-31, SPIE, Bellingham, WA. 1996

6. The system was christened ZEUS as an abbreviation for "Zap Every Usable Sample" thus continuing LLNL's long tradition of naming damage test systems (e.g. RASTER BLASTER, REPTILE, CHAMELEON, THOR, PLATO).

7. J. Hue, P. Garrec, J. Dijon, P. Lyan, " R-on-1 automatic mapping: a new tool for laser damage testing," in Laser Induced Damage in Optical Materials. SPIE Proceedings, Volume 2714, edited by H.E. Bennet et. al., 90-101, SPIE, Bellingham, WA. 1995

8. J. Hue, J. Dijon, P. Lyan, " The CMO YAG laser damage test facility," in Laser Induced Damage in Optical Materials. SPIE Proceedings, Volume 2714, edited by H.E. Bennet et. al., 102-113, SPIE, Bellingham, WA. 1995

9. K.E. Montgomery, F.P. Milanovich, "High laser damage threshold potassium dihydrogen phosphate crystals," Journal of Applied Physics, 68(8), 3979-3982, 1990.

10. L. Kamysheva, N. Burdanina, O. Zhukov, I. Gavrilova, A. Kovalenko, S. Savvinova, A. Savvinova, "Dielectric properties of KDP crystals containing additions of chromium," Soviet Physics - Crystallography 14(5), 816-818, 1970

11. A. Karguppikar, "Electronic Spectra of $\mathrm{CrO}_{4}^{2-}$ in KDP crystals," Physical status solidi (b) 64, K111-K114, (1974)

12. V. Bredikhin, N. Kiseleva, V. Korolikhin, "Applications of ultraviolet spectroscopy for the determination of impuritites in solutions and single crystals of $\mathrm{KH}_{2} \mathrm{PO}_{4}$ and $\mathrm{KD}_{2} \mathrm{PO}_{4}$," Inorganic Materials, 22(1), 94-96, 1986

13. E.B. Haugen, Probabilistic Mechanical Design, John Wiley and Sons, New York, 1980

14. S. Zacks, Introduction to Reliability Analysis, Springer Verlag, New York, 1992

15. M. Runkel, J. DeYoreo, W. Sell, D. Milam, 'Laser conditioning study of KDP on the Optical Sciences Laser using large area beams," to appear in these proceedings, SPIE, Bellingham, WA. 


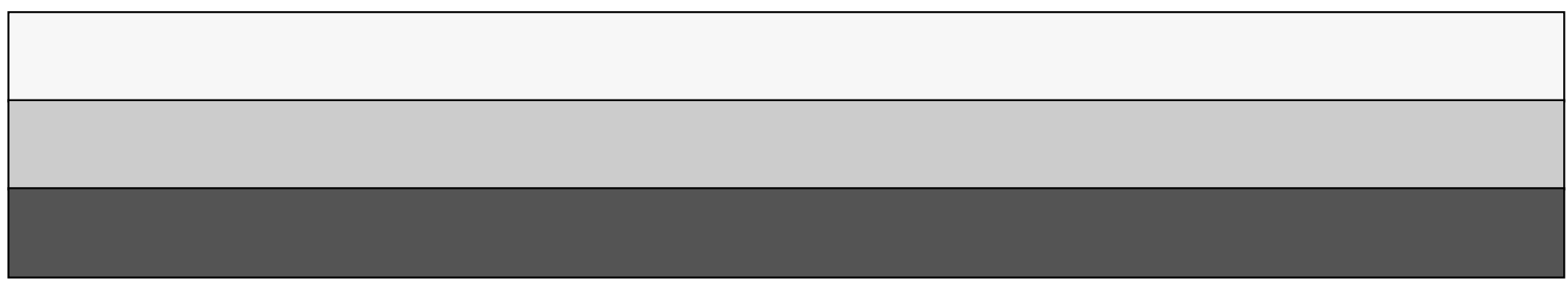

